\title{
Morpho-Physico-Chemical Characterization of Indian Wild Orange (Citrus indica Tanaka) grown in Nokrek Biosphere Reserve, Garo Hills, Meghalaya, India
}

\author{
S. Chetry*, C.P. Suresh, A.K. Chaurasiya and J.P. Lyngdoh \\ Department of Horticulture, North Eastern Hill University, Tura Campus, \\ Chasingre, Tura, West Garo Hills, Meghalaya-794002, India \\ *Corresponding author
}

\section{A B S T R A C T}

\section{Keywords}

Citrus indica, Endangered species, Citrus gene

Sanctuary,

Diversity,

Correlation

Article Info

Accepted:

12 December 2020

Available Online:

10 January 2021
Citrus indica is a wild endangered species of Citrus which is being protected and conserved at Nokrek Biosphere Reserve, West Garo Hills, Meghalaya, which has been designated as Citrus Gene Sanctuary. Therefore, villages near this reserve were selected with an objective to document the diversity of Citrus indica Tanaka so as to identify ideal accessions for different purposes. Samples were collected from 10 different locations and were evaluated for various qualitative and quantitative parameters. The analysis of variance for physical characters: tree height $(\mathrm{m})$, trunk girth $(\mathrm{cm})$, tree spread $(\mathrm{m})$, leaf length $(\mathrm{cm})$, leaf width $(\mathrm{cm})$, fruit length $(\mathrm{cm})$, fruit width $(\mathrm{cm})$, fruit weight $(\mathrm{g})$, number of fruit segment, juice content $(\%)$, rind thickness $(\mathrm{mm})$, seed length $(\mathrm{mm})$, seed width $(\mathrm{mm})$, seed weight $(\mathrm{g})$ and number of seeds per fruit; biochemical parameters: TSS $\left({ }^{\circ}\right.$ Brix $)$, total sugar (\%), reducing sugar (\%), non-reducing sugar $(\%)$, acidity $(\%), \mathrm{pH}$ and ascorbic acid $(\mathrm{mg} / 100 \mathrm{~g})$ were evaluated and significant variability for each trait among accession were observed. Based on fruit quality, accession from L-09SS was best and can be used for conservation, domestication, multiplication and breeding programmes. Correlation studies of 23 characters were evaluated and significant positive and negative correlation among some characters were observed.

\section{Introduction}

Indian wild orange (Citrus indica Tanaka) is locally known as Memang Narang in Garo language which means Ghost (Memang) Orange (Narang) (Singh, 1981) and in Khasi and Jaintia language it is called as Soh Kumphlair and $\mathrm{Sa}$ Kymphrai respectively (Upadhaya et al., 2016). It belongs to the family Rutaceae and sub family
Aurantioideae (Citroideae) and is one of the most primitive species believed to be the progenitor of today's cultivated Citrus species (Singh 1981). Tanaka (1928) was the first person to recognize Citrus indica, later Tanaka (1937) stated that 'It is really a wild citrus found in Nowgong district, Khasi hills and Manipur in Assam'. Bhattacharya and Dutta (1956) stated that the name Indian Wild Orange was retained because there was no 
vernacular name available for this species at the time. Citrus indica is a well-known wild species of Citrus, rich in nutritional content and has several medicinal benefits which has been utilized by the local people of the areas where it is found. The whole fresh fruit or juice of $C$. indica Tanaka can be utilized as an antidote for any kind of food poisoning. The dried fruit is also effective but should be used in higher doses. In addition, this fruit is reported to cure hypertension, snake bite, jaundice and smallpox. Juices are also used as energy drinks for persons suffering from fatigue and dehydration (Upadhaya et al., 2016).

The North-Eastern region is considered as the natural home and place of origin of a number of Citrus species. Citrus indica is native to North-Eastern Himalayas and found growing wild in Naga Hills, Nagaland, Kaziranga Reserve Forest, Assam and Garo Hills, Meghalaya (Singh 1981). Various surveys conducted to such areas indicated the presence of wild species of Citrus only in Tura range of Garo Hills, Meghalaya (Upadhyay and Sundriyal 1998; Singh and Singh 2003) but now it is also been found in different parts of North-East like Assam, Manipur, Arunachal Pradesh, Nagaland and Mizoram. The areas in Garo Hills, Meghalaya which falls in the buffer zone of Nokrek Biosphere Reserve has been designated as Citrus Gene Sanctuary (Singh 1981) and the germplasm of Indian Wild Orangeis conserved in-situat this sanctuary. This area is also famous for other wild Citrus species which provides a gene-pool for Citrus that are produced commercially. Tanaka (1937) and Bhattacharya and Dutta (1956) suggested that cultivation of Citrus indica hold no assurance for commercialization because of its fruits being small and practically inedible. The exploration and collection trip undertaken Malik et al., (2006) in the villages near Nokrek Biosphere Reserve Buffer Zone indicated the preliminary domestication and acclimatization process of $C$. Indica Tanaka near the Citrus Gene Sanctuary area. Plants of C. indica Tanaka growing outside the buff er zone area showed no flowering and fruiting in spite of healthy vegetative growth, thus indicating the lack of adaptation of this species to the man-made habitats which according to Zeven and de Wet (1982) is a complex phenomenon. In $C$. indica Tanaka, adaptations leading to domestication and to enable the species to enter into reproductive phase are still yet to be achieved. In addition, this hardy species found free from any pest and disease infestation in natural habitat, which can be a boost to Citrus industry, as in North-eastern states, Citrus decline is the major causing disease due to which Citriculture is in great threat, therefore, a promising rootstock resistant to decline and other pest and diseases has to be developed. Citrus indica Tanakamay prove to be a promising rootstock for commercial Citrus species once adapted to the varied climates, as it is confined to North-eastern states, their microclimate requirement is quite different than other Citrus species, as they are found in higher altitude, require cooler climate and found growing under tree shade with diffused sunlight. Therefore, adaptation of this species to various climatic factors has to be the first priority of researchers or breeders before utilizing its rootstock for commercial purposes. Commercialization of this species would also lead to its protection and utilization as a medicinal plant for which it is being valued by local people in Garo Hills.

Tremendous genetic diversity of wild and semi-wild Citrus germplasm of North-Eastern states has minimally been used for improvement programmes due to lack of their characterization. Systematic efforts for collection, characterization and conservation of genetic variability of $C$. indica Tanaka, are still lacking, therefore, considering all these 
aspects, study on Characterization of Citrus indica Tanaka was undertaken and the diversity of this species was documented for future use.

\section{Materials and Methods}

Ten promising accessions with three replications were selected from different places that are located nearby Nokrek Biosphere Reserve in the Garo Hills of Meghalaya. These places include Daribokgre, Mandalgre, Rongchek Manda, Rongchek Chambugong, Bandigre, Dura Ampanggre, Sakalgre, Baladinggre, Sasatgre and Dura Kalakgre. The data for Morphological Characters were taken according to the Minimal Descriptors of Agri-Horticultural Crops Part III: Fruit Crops (NBPGR, New Delhi). The fruits were collected during the month of December (2017) to March (2018) and these fruits were analyzed for morphological and biochemical characteristics and the experimental design followed was Completely randomized design (CRD).

The morphological parameters taken for characterization were tree habit, tree shape, branch density, leaf type, leaf persistency, leaf form, leaf or leaflet shape, leaf margin, petiole wing shape, inflorescence position, fruit shape, fruit base shape, fruit apex shape, fruit skin colour, fruit skin surface, mesocarp colour, pulp colour, pulp texture, juice colour, juice taste, juice aroma, seed shape, seed colour and some of the physical parameters were tree height $(\mathrm{m})$, tree girth $(\mathrm{cm})$, tree spread $(\mathrm{cm})$, leaf length $(\mathrm{cm})$, leaf width $(\mathrm{cm})$, fruit length $(\mathrm{cm})$, fruit width $(\mathrm{cm})$, fruit length to fruit width ratio, fruit weight $(\mathrm{g})$, rind thickness (mm), number of fruit segment, juice content $(\%)$, seed length $(\mathrm{m})$, seed width $(\mathrm{mm})$, seed weight $(\mathrm{g})$ and number of seed per fruit. The biochemical parameters were TSS, pH, acidity (Hortwitz, 1960), ascorbic acid
(Harris and Ray, 1935; Sadasivam and Balasubraminan, 1987), total sugar, reducing sugar and non-reducing sugar were analyzed following the method by Lane and Eynon (1923).

All the experimental data were subjected to Analysis of Variance (ANOVA) for one-way classification and was analyzed statistically using Agres Statistical Software Version, (c) 1994 Pascal Intl Software Solutions, Version 3.01and correlation coefficient was analyzed using SPSS software, Version 16.0.

\section{Results and Discussion}

The analysis of variance for all the characters indicates highly significant variation among the accessions collected from 10 different locations. All the accessions showed upright growth habits except the accessions from L02MD, L-06DA, L-09SS and L-10DK which showed compact growth habit. The tree shape observed was ellipsoid in shape; leaf showed simple leaf type, evergreen leaf persistency, sessile leaf form, lanceolate leaf or leaflet shape, dentate leaf margin; axillary inflorescence position; oblate fruit shape; truncate fruit base shape; truncate fruit apex shape; orange fruit skin colour; ovoid seed shape; cream seed colour in all the accessions. Smooth fruit skin surface was observed in all the accessions except for the accessions from L-02MD and L-06DA which were having longitudinal grooved and ridged fruit skin surface (Table 1).

The maximum tree height $(3.23 \mathrm{~m})$, trunk girth $(24.30 \mathrm{~cm})$ and tree spread $(\mathrm{EW}-2.67 \mathrm{~m})$ were recorded in accession from L-07SK and minimum tree height $(2.03 \mathrm{~m})$; trunk girth $(12.90 \mathrm{~cm})$ and tree spread (NS-1.57 $\mathrm{m}$ and EW-1.23 m) were obtained from L-08BL, whereas, the fruit size (fruit length-3.88 cm and fruit width-4.30 cm) and fruit weight $(36.50 \mathrm{~g})$ were observed maximum in 
accession from L-09SS and minimum fruit size (fruit length-2.11 cm and fruit width-2.66 $\mathrm{cm})$ were recorded from L-07SK while the minimum fruit weight (11.66 g) was observed in accession from L-04RC. The number of fruit segments ranged from 8.60 to 10.27 with maximum recorded from L-06DA and minimum from L-07SK. The juice content evaluated ranged from $19.84 \%$ to $37.97 \%$ with the highest juice percentage obtained from L-10DK and lowest from L-06DA. Rind thickness $(3.85 \mathrm{~mm})$, seed length $(12.71 \mathrm{~mm})$ and seed width $(8.89 \mathrm{~mm})$ were recorded highest from L-01DB and the lowest rind thickness $(1.80 \mathrm{~mm})$ and seed length (6.92 $\mathrm{mm}$ ) were obtained from L-05BD while lowest seed width $(6.10 \mathrm{~mm})$ was obtained from L-02MD. Seed weight ranged from $0.10 \mathrm{~g}-0.28 \mathrm{~g}$ with maximum from L-08BL and minimum from L-02MD, L-04RC and L06DA. The number of seeds ranged from 1.47 (L-09SS) to 12.53 (L-05BD).

The quality of citrus juice is an important economic factor for the citrus processing industry (Rouse, 2000). The juice/fruit is traditionally used by Garo tribes of Meghalaya for stomach disorder and other purposes by taking a small amount of fresh or stored juice in a cup of water with a pinch of salt (Upadhaya et al., 2016). Significantly, the highest TSS was obtained from L-02MD $\left(8.47^{\circ}\right.$ Brix) and the lowest from L-10DK $\left(5.33^{\circ}\right.$ Brix $)$, total sugar recorded were ranged from $1.69 \%$ to $3.24 \%$ in which maximum was obtained from L-09SS and the minimum from L-03RM, whereas reducing sugar obtained ranged from $1.16 \%$ to $2.67 \%$ and nonreducing ranged from $0.28 \%$ to $1.46 \%$ in which highest percentage of reducing and non-reducing sugar were recorded from L07SK and L-09SS respectively, highest titratable acidity was obtained from L-08BL (4.44\%) and lowest from L-03RM (1.97\%). These observations were also supported by the findings of Malik et al.,(2006) as their evaluated mean value for acidity was $2.33 \%$.
$\mathrm{pH}$ of fruit juices evaluated ranged from 2.58 to 2.93 with most acidic acquired from L01DB. Ascorbic acid evaluated in collected accessions varied significantly ranging from $6.82 \mathrm{mg} / 100 \mathrm{~g}$ to $37.92 \mathrm{mg} / 100 \mathrm{~g}$ with maximum recorded from L-05BD.

\section{Correlation coefficient}

Correlation studies of 23 characters of Citrus indica Tanaka were evaluated. This correlation analysis showed significant positive and negative correlation among some characters. As presented in Table 4 tree height showed positive and highly significant correlation with trunk girth, tree spread northsouth, tree spread east-west, total sugar and reducing sugar. Fruit weight exhibit positive correlation fruit length, fruit width, rind thickness, seed length, seed width, seed weight, juice content, total sugar and nonreducing sugar whereas it showed highly negative correlation with TSS, $\mathrm{pH}$ and ascorbic acid. Number of segment/fruits showed positive correlation with number of seeds/fruits, and exhibit negative correlation with sugars. Seed weight showed positive correlation with seed length, seed width, juice content, acidity and showed negative correlation with TSS, $\mathrm{pH}$ and ascorbic acid. Number of seeds/fruit exhibit highly significant positive correlation with only ascorbic acid and negative correlation with total sugar and non-reducing sugar. Juice content showed positive correlation with nonreducing sugar and exhibit highly negative correlation with TSS, $\mathrm{pH}$ and ascorbic acid. TSS showed positive correlation with $\mathrm{pH}$ and ascorbic acid. Acidity showed positive correlation with total sugar and reducing sugar. $\mathrm{pH}$ showed positive correlation with ascorbic acid. Ascorbic acid showed negative correlation with non-reducing sugar. Total sugar showed positive and highly significant correlation with reducing sugar and nonreducing sugar. 
Table.1 Qualitative traits of Citrus indica grown in Nokrek Biosphere Reserve Meghalaya, India

\begin{tabular}{|c|c|c|c|c|c|c|c|c|c|c|c|c|c|c|c|c|c|c|}
\hline Locations & $\begin{array}{c}\text { Tree } \\
\text { Habit }\end{array}$ & $\begin{array}{l}\text { Tree } \\
\text { Shape }\end{array}$ & $\begin{array}{l}\text { Branch } \\
\text { Density }\end{array}$ & $\begin{array}{l}\text { Leaf } \\
\text { Type }\end{array}$ & $\begin{array}{c}\text { Leaf } \\
\text { Persistency }\end{array}$ & $\begin{array}{l}\text { Leaf } \\
\text { Form }\end{array}$ & $\begin{array}{l}\text { Leaf or } \\
\text { Leaflet } \\
\text { Shape }\end{array}$ & $\begin{array}{c}\text { Leaf } \\
\text { Margin }\end{array}$ & $\begin{array}{c}\text { Inflorescence } \\
\text { Position }\end{array}$ & $\begin{array}{l}\text { Fruit } \\
\text { Shape }\end{array}$ & $\begin{array}{c}\text { Fruit } \\
\text { Base } \\
\text { Shape }\end{array}$ & $\begin{array}{l}\text { Fruit } \\
\text { Apex } \\
\text { Shape }\end{array}$ & $\begin{array}{c}\text { Fruit } \\
\text { Skin } \\
\text { Colour }\end{array}$ & $\begin{array}{c}\text { Fruit skin } \\
\text { surface }\end{array}$ & $\begin{array}{l}\text { Mesocarp } \\
\text { Colour }\end{array}$ & $\begin{array}{l}\text { Pulp } \\
\text { Texture }\end{array}$ & $\begin{array}{l}\text { Seed } \\
\text { Shape }\end{array}$ & $\begin{array}{l}\text { Seed } \\
\text { Colour }\end{array}$ \\
\hline L-01DB & Upright & Ellipsoid & Sparse & Simple & Evergreen & Sessile & Lanceolate & Dentate & Axillary & Oblate & Truncate & Truncate & Orange & Smooth & Pale Yellow & Tender & Ovoid & Cream \\
\hline L-02MD & Compact & Ellipsoid & Dense & Simple & Evergreen & Sessile & Lanceolate & Dentate & Axillary & Oblate & Truncate & Truncate & Orange & LG\&R & Pale Yellow & Tender & Ovoid & Cream \\
\hline L-03RM & Upright & Ellipsoid & Sparse & Simple & Evergreen & Sessile & Lanceolate & Dentate & Axillary & Oblate & Truncate & Truncate & Orange & Smooth & Pale Yellow & Tender & Ovoid & Cream \\
\hline L-04RC & Upright & Ellipsoid & Sparse & Simple & Evergreen & Sessile & Lanceolate & Dentate & Axillary & Oblate & Truncate & Truncate & Orange & Smooth & Pale White & Tender & Ovoid & Cream \\
\hline L-05BD & Upright & Ellipsoid & Sparse & Simple & Evergreen & Sessile & Lanceolate & Dentate & Axillary & Oblate & Truncate & Truncate & Orange & Smooth & Pale Yellow & Tender & Ovoid & Cream \\
\hline L-06DA & Compact & Ellipsoid & Medium & Simple & Evergreen & Sessile & Lanceolate & Dentate & Axillary & Oblate & Truncate & Truncate & Orange & LG\&R & Pale Yellow & Tender & Ovoid & Cream \\
\hline L-07SK & Upright & Ellipsoid & Sparse & Simple & Evergreen & Sessile & Lanceolate & Dentate & Axillary & Oblate & Truncate & Truncate & Orange & Smooth & Pale White & Tender & Ovoid & Cream \\
\hline L-08BL & Upright & Ellipsoid & Sparse & Simple & Evergreen & Sessile & Lanceolate & Dentate & Axillary & Oblate & Truncate & Truncate & Orange & Smooth & Pale Yellow & Tender & Ovoid & Cream \\
\hline L-09SS & Compact & Ellipsoid & Dense & Simple & Evergreen & Sessile & Lanceolate & Dentate & Axillary & Oblate & Truncate & Truncate & Orange & Smooth & Pale White & Tender & Ovoid & Cream \\
\hline L-10DK & Compact & Ellipsoid & Dense & Simple & Evergreen & Sessile & Lanceolate & Dentate & Axillary & Oblate & Truncate & Truncate & Orange & Smooth & Pale Yellow & Tender & Ovoid & Cream \\
\hline
\end{tabular}

LG\&R: Longitudinal grooved and ridges

Parameters: TH-Tree height; TG-Trunk girth; TSNS-Tree Spread-North South; TSEW-Tree Spread-East West; LL-Leaf Length; LW-Leaf Width; FL-Fruit Length; FW-Fruit Width; FWt. -Fruit Weight; RT-Rind Thickness; NOSg/F-Number of Segment/Fruit; SL-Seed Length; SW-Seed Width; SWt. -Seed Weight; NOS/F- Number of Seed/Fruit; JC-Juice Content; TSS-Total Soluble Solutes; AC-Acidity; AA-Ascorbic Acid; TS-Total Sugar; RS-Reducing Sugar; NRS-NonReducing Sugar 
Table.2 Physical traits of Citrus indica grown in Nokrek Biosphere Reserve Meghalaya, India

\begin{tabular}{|c|c|c|c|c|c|c|c|c|c|c|c|c|c|c|c|c|c|}
\hline \multirow[t]{2}{*}{ Locations } & \multirow{2}{*}{$\begin{array}{c}\text { Tree } \\
\text { height } \\
\text { (m) }\end{array}$} & \multirow{2}{*}{$\begin{array}{l}\text { Trunk } \\
\text { girth } \\
\text { (cm) }\end{array}$} & \multicolumn{2}{|c|}{ Tree spread (m) } & \multirow{2}{*}{$\begin{array}{l}\text { Leaf } \\
\text { length } \\
(\mathrm{cm})\end{array}$} & \multirow{2}{*}{$\begin{array}{c}\text { Leaf } \\
\text { width } \\
(\mathrm{cm})\end{array}$} & \multirow{2}{*}{$\begin{array}{l}\text { Fruit } \\
\text { length } \\
(\mathrm{cm})\end{array}$} & \multirow{2}{*}{$\begin{array}{l}\text { Fruit } \\
\text { width } \\
(\mathbf{c m})\end{array}$} & \multirow{2}{*}{$\begin{array}{c}\text { FL/F } \\
\text { W } \\
\text { (Ratio) }\end{array}$} & \multirow{2}{*}{$\begin{array}{c}\text { Fruit } \\
\text { weight } \\
\text { (g) }\end{array}$} & \multirow{2}{*}{$\begin{array}{c}\text { No. of } \\
\text { fruit } \\
\text { segment }\end{array}$} & \multirow{2}{*}{$\begin{array}{c}\text { Juice } \\
\text { content } \\
(\%)\end{array}$} & \multirow{2}{*}{$\begin{array}{c}\text { Rind } \\
\text { thickness } \\
(\mathbf{m m})\end{array}$} & \multirow{2}{*}{$\begin{array}{l}\text { Seed } \\
\text { length } \\
(\mathrm{mm})\end{array}$} & \multirow{2}{*}{$\begin{array}{c}\text { Seed } \\
\text { width } \\
(\mathrm{mm})\end{array}$} & \multirow{2}{*}{$\begin{array}{c}\text { Seed } \\
\text { weight } \\
\text { (g) }\end{array}$} & \multirow{2}{*}{$\begin{array}{c}\text { No. of } \\
\text { seeds/f } \\
\text { ruit }\end{array}$} \\
\hline & & & $\begin{array}{l}\text { North- } \\
\text { South }\end{array}$ & $\begin{array}{l}\text { East- } \\
\text { West }\end{array}$ & & & & & & & & & & & & & \\
\hline L-01DB & 2.47 & 15.20 & 2.83 & 2.27 & 7.17 & 3.10 & 3.61 & 4.13 & 0.87 & 33.79 & 10.07 & 31.51 & 3.85 & 12.71 & 8.89 & 0.24 & 10.33 \\
\hline L-02MD & 3.03 & 22.30 & 2.73 & 2.13 & 8.56 & 4.07 & 2.50 & 3.42 & 0.73 & 13.58 & 9.33 & 20.80 & 2.54 & 9.86 & 6.10 & 0.10 & 9.00 \\
\hline L-03RM & 2.43 & 15.07 & 2.20 & 1.37 & 9.99 & 4.44 & 2.43 & 3.37 & 0.72 & 12.76 & 10.20 & 27.62 & 2.14 & 11.36 & 7.03 & 0.11 & 8.53 \\
\hline L-04RC & 2.93 & 20.77 & 2.43 & 2.10 & 7.58 & 3.08 & 2.40 & 3.36 & 0.71 & 11.66 & 9.40 & 20.29 & 1.85 & 10.68 & 6.45 & 0.10 & 8.27 \\
\hline L-05BD & 2.90 & 21.53 & 2.50 & 2.20 & 9.37 & 4.24 & 2.59 & 3.45 & 0.75 & 15.91 & 9.93 & 23.14 & 1.80 & 6.92 & 7.09 & 0.14 & 12.53 \\
\hline L-06DA & 2.50 & 16.80 & 2.13 & 1.87 & 8.92 & 3.89 & 2.61 & 3.46 & 0.76 & 16.17 & 10.27 & 19.84 & 2.66 & 10.49 & 6.60 & 0.10 & 10.27 \\
\hline L-07SK & 3.23 & 24.30 & 2.77 & 2.67 & 8.74 & 3.73 & 2.11 & 2.66 & 0.79 & 19.14 & 8.60 & 23.26 & 2.20 & 10.60 & 7.21 & 0.15 & 6.47 \\
\hline L-08BL & 2.03 & 12.90 & 1.57 & 1.23 & 9.53 & 3.62 & 2.96 & 3.60 & 0.82 & 27.51 & 9.33 & 32.38 & 3.55 & 11.76 & 8.30 & 0.28 & 9.73 \\
\hline L-09SS & 3.13 & 23.53 & 2.83 & 2.57 & 9.27 & 4.10 & 3.88 & 4.30 & 0.90 & 36.50 & 8.93 & 36.43 & 3.76 & 11.13 & 6.94 & 0.18 & 1.47 \\
\hline L-10DK & 2.97 & 20.60 & 2.87 & 2.47 & 8.05 & 3.73 & 2.98 & 3.52 & 0.85 & 30.15 & 9.80 & 37.97 & 2.62 & 11.06 & 7.86 & 0.19 & 10.13 \\
\hline Mean & 2.76 & 19.30 & 2.48 & 2.08 & 8.71 & 3.80 & 2.80 & 3.53 & 0.79 & 21.71 & 9.58 & 27.32 & 2.69 & 10.65 & 7.24 & 0.15 & 8.67 \\
\hline S.Ed & 0.16 & 1.94 & 0.19 & 0.24 & 0.838 & 0.29 & 0.13 & 0.09 & & 2.91 & 0.58 & 4.21 & 0.29 & 0.67 & 0.50 & 0.02 & 1.03 \\
\hline C.D(.05) & 0.34 & 4.05 & 0.40 & 0.51 & 1.74 & 0.62 & 0.27 & 0.18 & & 6.08 & 1.21 & 8.78 & 0.62 & 1.40 & 1.04 & 0.04 & 2.15 \\
\hline
\end{tabular}

Table.3 Biochemical traits of Citrus indica grown in Nokrek Biosphere Reserve Meghalaya, India

\begin{tabular}{|c|c|c|c|c|c|c|c|c|}
\hline Location & $\begin{array}{c}\text { TSS } \\
\left({ }^{\circ} \text { Brix }\right)\end{array}$ & $\begin{array}{c}\text { Total sugar } \\
(\%)\end{array}$ & $\begin{array}{l}\text { Reducing } \\
\text { Sugar }(\%)\end{array}$ & $\begin{array}{l}\text { Non-Reducing } \\
\text { Sugar }(\%)\end{array}$ & $\begin{array}{l}\text { Acidity } \\
(\%)\end{array}$ & pH & $\begin{array}{l}\text { Asc. Acid } \\
(\mathrm{mg} / \mathbf{1 0 0 g})\end{array}$ & $\begin{array}{c}\text { TSS/ACID } \\
\text { Ratio }\end{array}$ \\
\hline L-01DB & 5.40 & 2.29 & 1.65 & 0.61 & 3.20 & 2.58 & 12.88 & 0.71 \\
\hline L-02MD & 8.47 & 2.35 & 1.83 & 0.50 & 4.27 & 2.80 & 35.52 & 0.55 \\
\hline L-03RM & 6.80 & 1.69 & 1.40 & 0.28 & 1.97 & 2.73 & 24.96 & 0.85 \\
\hline L-04RC & 7.13 & 2.21 & 1.62 & 0.57 & 2.69 & 2.93 & 27.36 & 0.82 \\
\hline L-05BD & 7.40 & 2.64 & 2.08 & 0.54 & 3.58 & 2.63 & 37.92 & 0.73 \\
\hline L-06DA & 6.73 & 1.76 & 1.16 & 0.57 & 3.37 & 2.83 & 30.24 & 0.52 \\
\hline L-07SK & 5.93 & 3.06 & 2.67 & 0.37 & 3.63 & 2.70 & 15.09 & 0.84 \\
\hline L-08BL & 5.60 & 2.12 & 1.64 & 0.46 & 4.44 & 2.67 & 11.41 & 0.47 \\
\hline L-09SS & 6.13 & 3.24 & 1.71 & 1.46 & 3.54 & 2.67 & 6.80 & 0.91 \\
\hline L-10DK & 5.33 & 2.20 & 1.74 & 0.43 & 3.71 & 2.71 & 7.73 & 0.59 \\
\hline Mean & 6.49 & 2.35 & 1.75 & 0.57 & 3.40 & 2.72 & 20.99 & 0.69 \\
\hline S.Ed & 0.55 & 0.05 & 0.03 & 0.03 & 0.21 & 0.10 & 2.68 & \\
\hline C.D(.05) & 1.16 & 0.11 & 0.07 & 0.07 & 0.45 & 0.22 & 5.59 & \\
\hline
\end{tabular}

1915 
Table.4 Correlation coefficient among 23 characters of Citrus indica Tanaka

\begin{tabular}{|c|c|c|c|c|c|c|c|c|c|c|c|c|c|c|c|c|c|c|c|c|c|c|c|}
\hline $\begin{array}{r}\text { Paramet } \\
\text { ers }\end{array}$ & TH & TG & TSNS & TSEW & LL & LW & FL & FW & FWt. & RT & NOSg/F & SL & SW & SWt. & $\begin{array}{r}\text { NOS/ } \\
\text { F } \\
\end{array}$ & JC & TSS & AC & PH & AA & TS & RS & NRS \\
\hline TH & 1 & $.965^{* *}$ & $.746^{* *}$ & $.804^{* * *}$ & -.227 & .024 & -.111 & -.189 & -.009 & -.253 & -.241 & -.233 & -.302 & $-.375^{*}$ & $-.360^{*}$ & -.067 & .168 & .049 & .176 & .067 & $.618^{* *}$ & $.532^{* *}$ & .277 \\
\hline TG & & 1 & $.673^{* *}$ & $.750^{* * *}$ & -.158 & .068 & -.135 & -.202 & -.003 & -.257 & -.281 & -.299 & $-.341^{*}$ & $-.341^{*}$ & $-.358^{*}$ & -.11 & .207 & .133 & .109 & .083 & $.661^{* *}$ & $.559^{* * *}$ & $.308^{*}$ \\
\hline TSNS & & & 1 & $.838^{* *}$ & $-.313^{*}$ & .011 & .202 & .111 & .231 & .002 & -.153 & -.019 & .045 & -.102 & -.271 & .181 & -.019 & -.035 & -.103 & -.142 & $.519^{* *}$ & $.404^{*}$ & .283 \\
\hline TSEW & & & & 1 & $-.336^{*}$ & -.063 & .149 & .004 & .253 & .014 & -.217 & -.093 & .044 & -.042 & -.291 & .165 & -.06 & .135 & -.09 & -.192 & $.640^{* *}$ & $.494^{* *}$ & $.354^{*}$ \\
\hline LL & & & & & 1 & $.623^{* *}$ & -.141 & -.1 & -.116 & -.198 & .061 & -.189 & -.235 & -.027 & -.066 & .14 & .121 & -.074 & -.105 & .016 & .003 & -.026 & .037 \\
\hline LW & & & & & & 1 & -.128 & -.142 & -.24 & -.277 & -.07 & $-.368^{*}$ & -.279 & -.217 & -.1 & .031 & $.369^{*}$ & -.032 & -.165 & .218 & .04 & .014 & .043 \\
\hline FL & & & & & & & 1 & $.910^{* *}$ & $.843^{* *}$ & $.776^{* *}$ & .065 & $.422^{*}$ & $.460^{* *}$ & $.607^{* *}$ & $-.315^{*}$ & $.611^{* *}$ & $-.396^{*}$ & .146 & $-.393^{*}$ & $-.565^{* *}$ & .254 & -.286 & $.713^{* *}$ \\
\hline FW & & & & & & & & 1 & $.665^{* *}$ & $.685^{* *}$ & .185 & $.306^{*}$ & .301 & $.420^{*}$ & -.2 & $.472^{* *}$ & -.218 & .01 & -.286 & $-.324^{*}$ & .065 & $-.501^{* *}$ & $.689^{* *}$ \\
\hline FWt. & & & & & & & & & 1 & $.752^{* *}$ & -.041 & $.501^{* *}$ & $.565^{* *}$ & $.733^{* *}$ & -.264 & $.725^{* *}$ & $-.645^{* *}$ & .285 & $-.460^{* *}$ & $-.801^{* *}$ & $.360^{*}$ & -.006 & $.538^{* *}$ \\
\hline RT & & & & & & & & & & 1 & .025 & $.564^{* *}$ & $.522^{* *}$ & $.649^{* *}$ & -.297 & $.532^{* *}$ & $-.516^{* *}$ & $.375^{*}$ & -.3 & $-.554^{* *}$ & .145 & -.231 & $.489^{* * *}$ \\
\hline $\mathrm{NOSg} / \mathrm{F}$ & & & & & & & & & & & 1 & .131 & .134 & -.045 & $.499^{* *}$ & .082 & -.035 & -.29 & -.045 & .23 & $-.543^{* *}$ & $-.487^{* *}$ & -.222 \\
\hline SL & & & & & & & & & & & & 1 & $.537^{* *}$ & $.468^{* *}$ & -.24 & $.397^{*}$ & $-.499^{* *}$ & -.139 & -.035 & $-.622^{* *}$ & -.212 & $-.311^{*}$ & .058 \\
\hline SW & & & & & & & & & & & & & 1 & $.761^{* *}$ & .181 & $.553^{* *}$ & $-.666^{* *}$ & .142 & $-.475^{* *}$ & $-.515^{* *}$ & -.036 & .025 & -.08 \\
\hline SWt. & & & & & & & & & & & & & & 1 & .013 & $.598^{* *}$ & $-.603^{* *}$ & $.363^{*}$ & $-.589^{* *}$ & $-.673^{* *}$ & .144 & .07 & .131 \\
\hline NOS/F & & & & & & & & & & & & & & & 1 & -.158 & .047 & .021 & -.158 & $.434^{* *}$ & $-.586^{* *}$ & -.147 & $-.687^{* * *}$ \\
\hline JC & & & & & & & & & & & & & & & & 1 & $-.707^{* *}$ & .143 & $-.499^{* *}$ & $-.727^{* * *}$ & .156 & -.078 & $.324^{*}$ \\
\hline TSS & & & & & & & & & & & & & & & & & 1 & -.058 & $.377^{*}$ & $.725^{* *}$ & -.077 & -.039 & -.071 \\
\hline AC & & & & & & & & & & & & & & & & & & 1 & -.124 & -.098 & $.334^{*}$ & $.308^{*}$ & .126 \\
\hline PH & & & & & & & & & & & & & & & & & & & 1 & $.311^{*}$ & -.222 & -.203 & -.088 \\
\hline AA & & & & & & & & & & & & & & & & & & & & 1 & -.3 & -.086 & $-.342^{*}$ \\
\hline TS & & & & & & & & & & & & & & & & & & & & & 1 & $.735^{* *}$ & $.599^{* *}$ \\
\hline RS & & & & & & & & & & & & & & & & & & & & & & 1 & -.102 \\
\hline NRS & & & & & & & & & & & & & & & & & & & & & & & 1 \\
\hline
\end{tabular}


Figure.1 Trees variability in Citrus indica collected from nearby areas of Nokrek Biosphere Reserve, Meghalaya
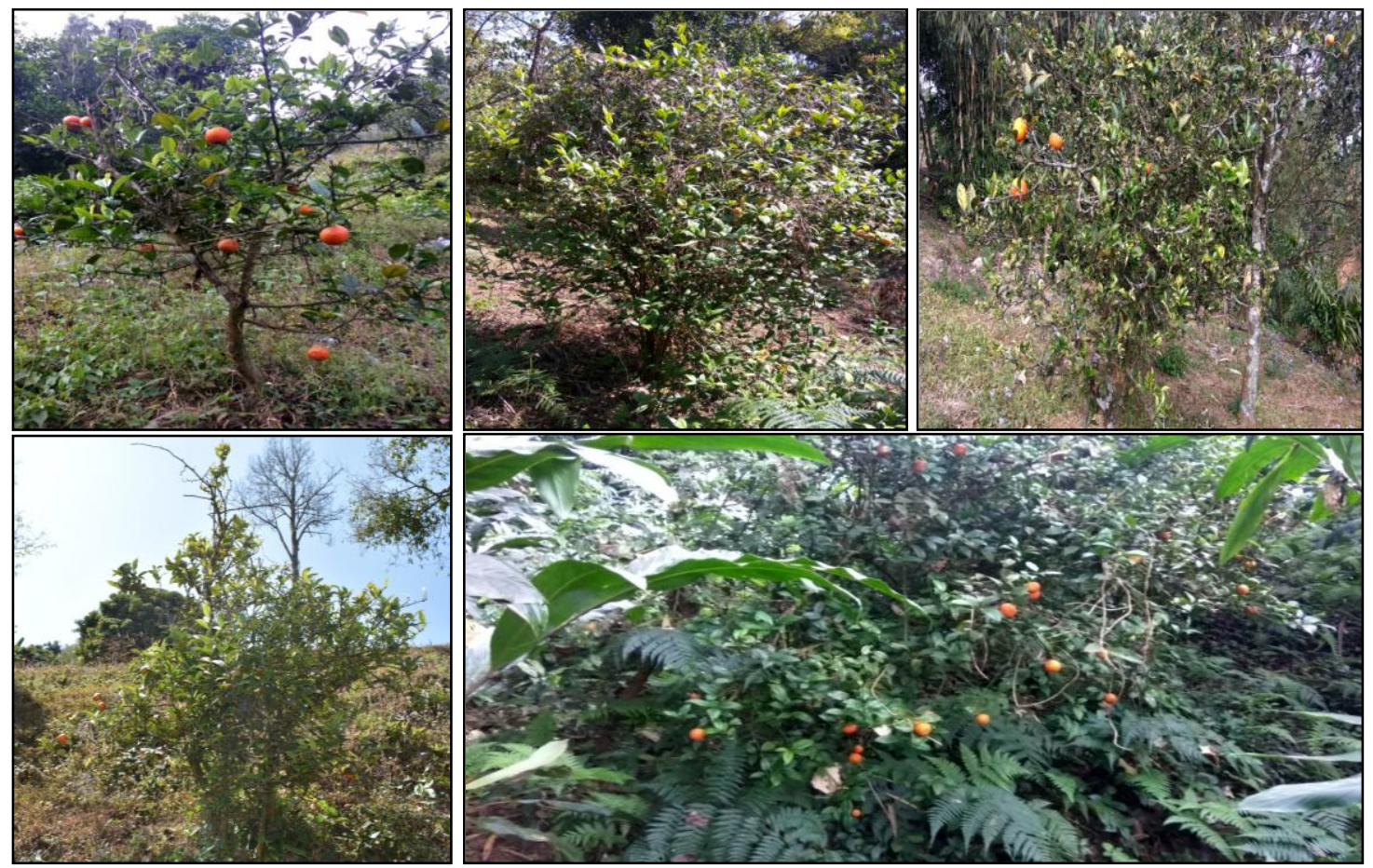

Figure 2: Fruits variability in Citrus indica collected from nearby areas of Nokrek Biosphere Reserve, Meghalaya

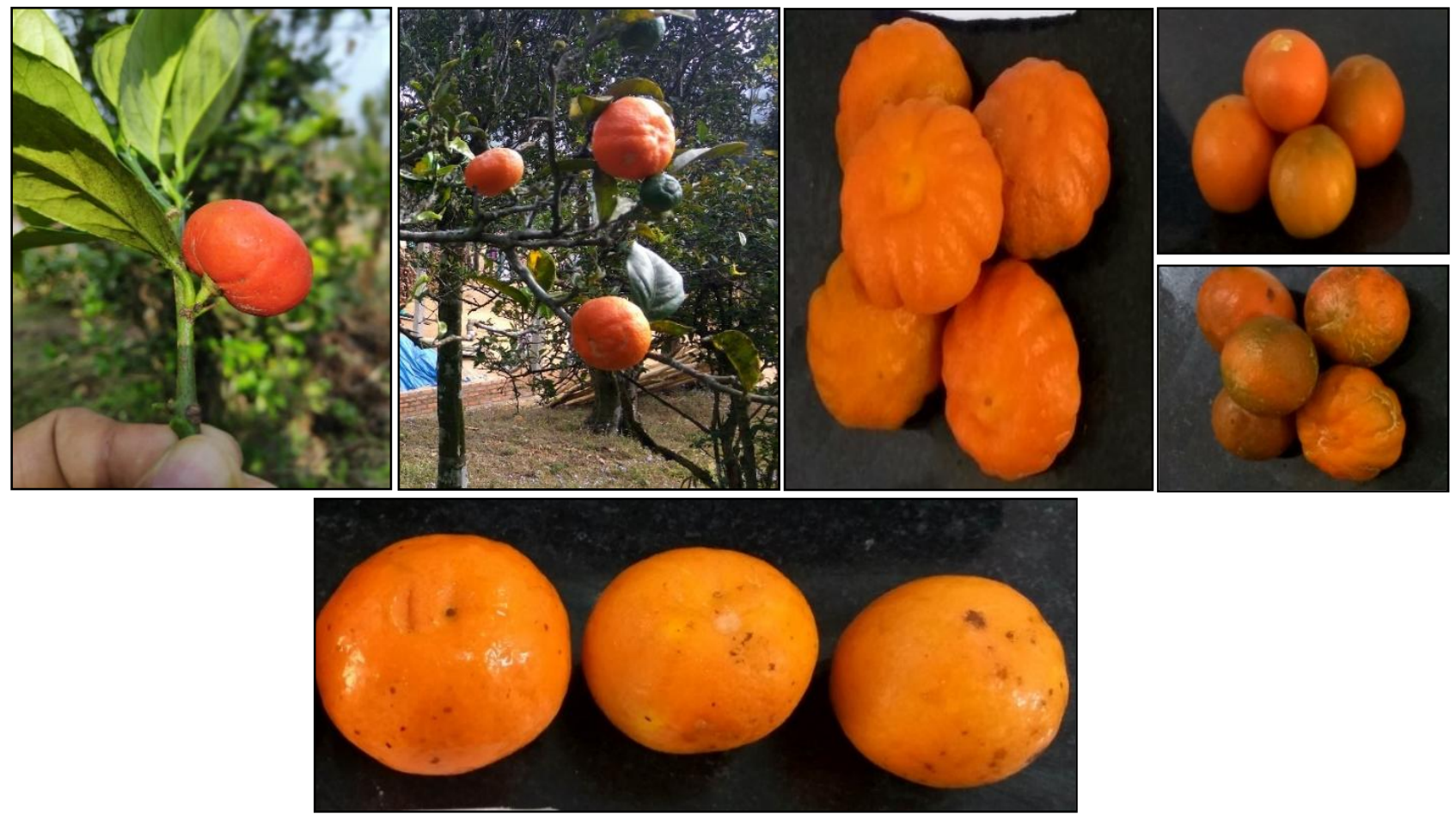


Citrus indica is one of the most important species of Citrus which is believed to be an ancestor of today's edible Citrus. This fruit is found only in a few pockets of North-eastern India such as Meghalaya, Assam, Arunachal Pradesh, Mizoram and Nagaland. Apart from being popularly known as ancestor of Citrus, Citrus indica Tanaka is also an important species in traditional medicine as it is used for different purposes, like, the fruit is used to treat jaundice and other related stomach disorder of humans as well domestic animals. The fruit along with the peel is also used by the Garo tribe as an antidote for snake bite. For curing smallpox, powdered form as well as raw fruit as whole can be used (Upadhaya et al., 2016). In this research, significant differences have been observed from 10 different locations selected for study. The present data as reflected in Table 1 revealed that all the qualitative parameters showed more or less the same appearance except for few, but the fruit skin surface of accession from L-02MD and L-06DA showed longitudinal grooved and ridges skin surface and the rest showed smooth skin surface. Therefore, the recorded variation in the fruit skin surface reveal that thereare varietal differences within the species and thus further investigation may lead to the development of new varieties.

Elaborate surveys made to different locations revealed that Citrus indica grows well under shade and is mostly found growing wild in the jungle and also been observed growing in the home garden of the farmers. Better plant growth was observed in accession from L07SK and L-09SS along with the fruit weight which was observed highest in accession from L-09SS followed by L-01DB though the fruits of L-01DB and L-10DK are small in appearance. The difference in their fruit weight was recorded to be $3 \mathrm{~g}$ and $6 \mathrm{~g}$ less than the fruit weight of accession from L-09SS respectively. Very less differences in fruit weight had been observed if we look at to the size of the fruits, which can be due to the increase in percentage of juice content (31.51\% and $37.97 \%$ respectively) and the reason behind the good growth of accession from L-09SS may be due to its surrounding climate as it was found growing under the shade of Mandarin (Citrus reticulata), Boldubak (Kydia calycina Roxb.), Bauhinia variegata whereas Elaichi was found to be cultivating by the farmer which creates a congenial environment for its growth and development. It was observed that accession from L-01DB though did not found growing under the shade, it grows well and found fruiting throughout the year as this village have microclimatic condition similar to that of Gene Sanctuary. The number and size of segments have a bearing influence on internal quality of fruit. Budathoki (2004) opined that lesser the numbers of segments, the thicker is each segment, and hence more pulp in the fruit. Number of fruit segments varied from 8.60 to 10.27 which can also be one of the reasons for the increase in fruit weight. Such variability in the number of segments may be attributed to cross-compatibility between genotypes affecting parents of separate fruit segment numbers. Simultaneous flowering and fruiting were observed in accession from L-01DB during the collection period which made our collection easier due to its availability throughout the year but the same was not observed from other locations. Early September to January was observed to be the flowering period and fruiting started from Mid-October to February.Plants of $C$. indica Tanaka growing outside the buffer zone area showed no flowering and fruiting in spite of healthy vegetative growth, thus indicating the lack of adaptation of this species to the manmade habitats which according to Zeven and de Wet (1982) is a complex phenomenon. In C. indica Tanaka, adaptations leading to domestication and to enable the species to enter into reproductive phase are still yet to be achieved. 
Concentration of biochemical compositions such as TSS, acids and ascorbic acid content are the main internal factors that determine the quality of citrus in the world (BabazadehDarjazi et al., 2009; Antonucci et al., 2011). The citrus accessions revealed significant variation in internal quality characteristics. Total soluble solids ranged from $5.33^{\circ}$ Brix to $8.47^{\circ} \mathrm{Brix}$ with highest TSS recorded from L$02 \mathrm{MD}$, acid content ranged from $1.97 \%$ to $4.44 \%$, taste from less sour to acrid sour with highest acid content from accession of $\mathrm{L}$ 08BL which are slightly less than lime and lemon as are considered to have higher acid content than the other citrus fruits which may go up to $6 \%$ and $8 \%$ respectively. The TSS:acid ratio is an important trait determining the quality of citrus fruits. The content of total soluble solids (TSS) and acids as well as the fine balance between them plays a significant role in depicting internal quality of citrus fruits. In the present study, the TSS/acid ratio of Citrus indica varied from 0.477 to 0.915 with highest TSS: Acid ratio observed from L-09SS, the increase in TSS: acid ratio is due to an increase in TSS. The blend of TSS and acid gives the characteristics taste and flavour. The values of ascorbic acid obtained ranged from 6.80 $\mathrm{mg} / 100 \mathrm{~g}$ to $37.92 \mathrm{mg} / 100 \mathrm{~g}$ which can serve as good anti-oxidant properties. This variation may be attributed to the fact that the amount of ascorbic acid content of citrus fruits is rarely constant but differs with several factors like tree position; climatic conditions; period of maturation and various diversity of the citrus fruits; nitrogen content as well as temperature (Holcombe, 1992). Though sugar in other Citrus species like $C$. reticulata, $C$. sinensis etc. tends to increase with maturity and acid content decreases but according to the present study it is found that the acid content of all the accessions of Citrus indica is recorded more than total sugar content. Total sugar ranged from $1.69 \%$ to $3.24 \%$ with highest sugar content observed from L-09SS which can be said that the fruits are acidic in nature. Reducing sugar ranged from $1.16 \%$ to $2.67 \%$ with highest recorded from L-06DA whereas non reducing sugar ranged from $0.28 \%$ to $1.46 \%$ with highest observed from L-09SS. Reducing sugar of Citrus indica is observed to be very low, the two sugars i.e., glucose and fructose are referred to as reducing sugar (Zoecklein et al., 1990). Fructose, also called as fruit sugar is the sweetest sugar found naturally in fruits and therefore the low content of reducing sugar in Citrus indica makes it sour and more acidic in taste. The quality of fruit is an important factor which determines the popularity of this fruit among the consumers. The taste of Citrus indica ranges from sour to acid sour which is not preferable by the consumers but due to its medicinal properties, being ancestors of today's edible citrus and its confinement to North-eastern states of India, it is being conserved and Citrus Gene Sanctuary has been established under Nokrek Biosphere reserve to preserve in its natural habitat.

Correlation analysis shows the interrelationships between different characters and helps in effective identification of potential accessions for any improvement program. Fruit weight showed maximum correlation with fruit length followed by rind thickness, juice content, seed weight and fruit width. Negative correlation of fruit weight with TSS and ascorbic acid was obtained which revealed that high fruit quality under natural condition can be obtained as the fruit weight of most of the accessions are less which ultimately gives high TSS and ascorbic acid content. Two important characters like rind thickness and acidity showed positive correlation whereas rind thickness was negatively correlated with TSS and ascorbic acid indicating scope with breeding fruits with thin rind and less acid in association with high TSS and high ascorbic acid content. This 
association revealed that improvement of Citrus indica for thin rind and less acid may also bring about improvement in TSS and ascorbic acid. Positive correlation between number of segment/fruit and number of seed/fruits revealed that with increase in number of segment/fruits, number of seed/fruits also increases which is an obvious result. Positive correlation was observed between TSS and ascorbic acid whereas TSS was negatively correlated with acidity which could be an important character for selection of an improved cultivar with high TSS and ascorbic acid along with less acidity which are a key factor that determine the quality of fruits.As there is no doubt, that in order to select the best accessions, the fruit characters are an important factor that determine the quality and preferences of the consumers. The quality of citrus fruits depends on several factor like its juice content, TSS, acidity level and ascorbic acid content which revealed quite a good result in this present research. The results are in support with the findings of Khandavi (2012) who reported positive correlation of fruit weight with fruit length and breadth in Sweet oranges and Kagzi lime. Our finding is also been supported by Rehman et al., (1983) where they reported positive correlation between fruit weight and juice content but werein contrary to our finding where they reported negative correlation between juice content and acidity and positive correlation between fruit weight and TSS. However, Pingle (2011) reported negative correlation between TSS and acid in Kagzi lime which is in support with our findings.

In conclusion, the various accessions of Citrus indica Tanaka varied significantly with respect to morphological and biochemical properties and from the above findings it can be concluded that the accession from Sasatgre (L-09SS) was the best on the basis of physical parameters followed by Daribokgre (L-
01DB). Biochemical parameters of fruits like TSS:acid ratio and ascorbic acid content are important traits that determine the quality of citrus fruits. Ascorbic acid content was found highest in the accession from Bandigre (L05BD) followed by Daribokgre (L-01DB) and TSS was highest in accession from Mandalgre (L-02MD) and Bandigre (L-05BD). The significant and positive correlation was obtained from different characters like fruit weight with rind thickness, seed weight and juice content. Important characters like rind thickness showed positive correlation with juice content and acidity; TSS and ascorbic acid showed positive correlation but showed negative correlation with acidity. Therefore, this result revealed positive and negative correlation between different important characters and hence breeding programme should be taken in accordance with the desired character.

This study reveals that the rich diversity of citrus is not only found in an interspecies comparison but also within a single species as well. Based on the use of Citrus indica fruits in the Garo Hills region of Meghalaya, we were able to identify the accessions that are ideal for different purposes based on their morphological and biochemical traits. Understanding the diversity of Citrus indica in this region will aid in identification and conservation efforts that will benefit the community in exploiting this species in a more efficient manner.

These findings also serve as a significant reference for selection of suitable accessions for Citrus breeding programmes with the accession having the highest extent of variation. Moreover, the findings in this study can also serve as a primary source for future research efforts focussing on Citrus indica and its potential use in medicine that goes beyond the traditional knowledge of the Garo tribe. 


\section{Acknowledgement}

We thank the Pro-Vice Chancellor Prof. G. Singaiah for his encouragement and support and we are also grateful to the Department of Horticulture, North Eastern Hill University, Tura Campus for providing research facilities. Special thanks to Smt. N.R.D. Marak, MFS Divisional Forest Officer, Ease and West Garo Hills, Wildlife Division, Tura, Meghalaya for permitting us to collect the samples from reserved area.

\section{References}

Antonucci F, Pallottino F, Paglia G, Palma A, Aquino SD, Menesatti P. (2011). Nondestructive estimation of mandarin maturity status through portable VISNIR spectrophotometer. Food and Bioprocess Technology 4(5): 809-813. https://doi.org/10.1007/s11947-0100414-5.

Babazadeh Darjazi B, Rustaiyan A, Talaei A, Khalighi A, Golein B, Hayatbakhsh E, Taghizad R (2009). The effects of rootstock on the volatile flavour components of page mandarin $[(C$. reticulata var Dancy, $C$. paradisi var Dancan) C. clemantina] juice and peel. Iranian Journal of Chemistry and Chemical Engineering 28(2): 99-111.

Feldsine P, Abeyta C, Andrews WH (2002). AOAC International methods committee guidelines for validation of qualitative and quantitative food microbiological official methods of analysis. Journal of AOAC International 85(5):1187-1200.

Harris LJ, Ray SN (1935). Biochemical methods. Lancet 1:462-463.

Holcombe GD (1992) Fruit growth and development, Applied Botany, 1st ed. Living stones Publisher, Churchill. pp46.

Hossain M, Haque A (1977). Studies on the physical characteristics of jackfruit Bangladesh Hort. 5(1): 9-14.

Hortwitz W (1960). Official and tentative methods of analysis. Association of the Official Agriculture Chemist, Washington, DC 9: 320-341.

http://www.catalogueoflife.org/col/details/spe cies/id/097ce80ec5a9ac7a809770793fe8 $72 \mathrm{f} 9$

http://westgarohills.gov.in/profile.html https://whc.unesco.org/en/tentativelists/6356/

Khandavi R S (2012). Survey for selection of superior Kagzi lime (Citrus aurantifolia Swingle) strains in Beed district. Dissertation, M.K.V., Parbhani, M.S. (India).

Lane JH, Eynon L (1923). Methods for determination of reducing and nonreducing sugars. Journal of Science 42:32-37.

Mahajan RK, Gangopadhyay KK, Gunjeet Kumar DV, Srivastava U, Gupta PN (2002). Minimal descriptors of AgriHorticultural crops. Part III: Fruit Crops. New Delhi: National Bureau of Plant Genetic Resources.

Malik SK, Chaudhury R, Dhariwal OP, Kalia K, Rajwant (2006). Collection and characterization of Citrus indica Tanaka and $C$. macroptera Montr: wild endangered species of Northeastern India. Genetic Resources and Crop Evolution 53:1485-1493. https://doi.org/10.1007/s10722-0057468-7.

Marboh ES (2014). Morphological Characterization of Citrus Genotypes. Dissertation, Division of Fruits and Hort. Technology, Indian Agricultural Research Institute New Delhi.

Pingle SN (2011). Survey for selection of superior Kagzi lime (Citrus aurantifolia Swingle) strains in Latur district. Dissertation, M.K.V., Parbhani, M.S. (India).

Rabha A, Wangchu L, Singh B (2013). 
Studies on Genetic Diversity of citrus in East Siang District of Arunachal Pradesh. International Journal of Agriculture, Environment and Biotechnology, 6(1): 131-137.

Rehman S, Ahmad A, Ahmad I and Ghaffoor. (1983). Quantitative aspects of various cultivars of sweet oranges (Citrus sinensis). Pak J Agric Res 4:22-28.

Rouse RE (2000). Citrus fruit quality and yield of six Valencia clones on 16 rootstocks in the Immokalee Foundation Grove. Proceedings of the Florida State Horticultural Society113: 112-114.

Sadasivam S, Balasubramanian T. (1987). Practical manual in biochemistry. Tamil Nadu Agricultural University, Coimbatore, India 14.

Singh B (1981). Establishment of First Gene Sanctuary for Citrus in Garo Hills. Concept Publishing Company, New Delhi.

Singh IP, Singh S (2003). Exploration, collection and mapping of citrus genetic diversity in India. Technical BulletinNo.7, National Research Centre for Citrus, Nagpur.

Syamal MM, Mishra KA (1989). Physicochemical analysis of some important mango cultivars of Bihar. Acta
Horticulturae, 231:149-151.

Tanaka T (1928). On certain new Species of citrus. StudiaCitrological2: 155-164.

Tanaka T (1937). Citrus fruits of India. Allahabad Farmer 2:53-57.

Upadhyay RC, Sundriyal RC (1998). Crop gene pools in the Northeast Indian Himalayas and threats. In: Pratap T. and Sthapit B. (eds), Managing Agrobiodiversity-Farmers Changing Perspective and Institutional Responses in the Hindu Kush-Himalayan Region. ICIMOD and IPGRI, Kathmandu, Nepal167-173.

Upadhaya A, Chaturvedi SS, Tiwari BK (2016). Utilization of wild Citrus by Khasi and Garo tribes of Meghalaya. Indian Journal of Traditional Knowledge, 15(1):121-127.

Woodford RC (2008). Citrus Classification. Biotech Books: 98-102.

Zeven AC, de Wet JMJ (1982). Dictionary of Cultivated Plants and Their Regions of Diversity. Centre Publishing and Documentation, Wageningen, The Netherlands.

Zoecklein BW, Fugelsang KC, Gump BH, Nury FS (1990). Carbohydrates: Reducing Sugars. In: Production Wine Analysis. Springer, Boston, MA.

\section{How to cite this article:}

Chetry, S., C.P. Suresh, A.K. Chaurasiya and Lyngdoh, J.P. 2021. Morpho-Physico-Chemical Characterization of Indian Wild Orange (Citrus indica Tanaka) grown in Nokrek Biosphere Reserve, Garo Hills, Meghalaya, India. Int.J.Curr.Microbiol.App.Sci. 10(01): 1910-1922. doi: https://doi.org/10.20546/ijcmas.2021.1001.223 